\title{
Performance Evaluation of FBMC for Future Communication
}

\author{
Dr. Shankaraiah ${ }^{* 1}$, Tejas K B ${ }^{2}$ \\ "Professor, Department of ECE, SJCE, JSS STU, Mysuru, Karnataka, India \\ ${ }^{2} \mathrm{PG}$ Scholar, NIE(MTech), Department of ECE, SJCE, JSS STU, Mysuru, Karnataka, India
}

\begin{abstract}
Article Info

Volume 8, Issue 4

Page Number: 199-206

Publication Issue :

July-August-2021

\section{Article History}

Accepted : 20 July 2021

Published: 25 July2021

The current mobile telecommunication system is grew in a massive way, and in upcoming days requires a good supervision and enactment evaluation in addition to analysis. Filter-Bank-Multi-Carrier (FBMC) stands a method of multicarrier cadence method that is been extremely used for high date rate cellular network system as well as wireless systems. This entails a supple distribution of the obtainable time-frequency possessions, which is problematic over conservative Orthogonal Frequency Division Multiplexing (OFDM). Consequently, alterations of OFDM, such as windowing otherwise filtering, develop essential. The intention of current investigation exertion grounded on mobile expertise remains to upsurge the bandwidth in lieu of altogether users, huge bandwidth, further effectual also easily wieldy also continual unchanging connectivity. The projected paper benevolences, a coalescing outline, and discussion also performance estimation of FBMC also associate it toward OFDM grounded patterns. We develop that several antennas also channel estimate, two of the chief defies allied by FBMC, be able to be proficiently dealt through.

Keywords : FBMC, 5G, OFDM, Mobile Communication, bandwidth, modulation
\end{abstract}

\section{INTRODUCTION}

The Fourth generation mobile net is basically the development for the $3 G$ revolution by additional transmission rapidity besides presidencies proposals of the $3 \mathrm{G}$. The $4 \mathrm{G}$ which develops the capability to interface by means of wire line and mobile network also that convey numerous hypermedia also data transverse the sphere. $4 \mathrm{G}$ remains a intangible charter which necessities high rapidity wireless net to transmission data. The $5 \mathrm{G}$ nets stance going towards be a colossal net entity also determination be erected round a mismatch of known : 2G, LTE-A, 3G, LTE, M2M, Wi-Fi, etc. Over further confrontations, $5 \mathrm{G}$ determination be intended to provision a diversity of requests such by way of the IoT, linked wearable's, amplified realism besides immersive speed. 
Upcoming mobile structures resolution remain extremely varied also branded thru a huge variety of conceivable usage cases, existing on or after improved Mobile Comprehensive new terminated improved Mechanism Type Communication to Extreme Consistent Squat expectancy application in vehicular communication. To proficiently sustenance such varied usage circumstances, we essential a supple distribution of the obtainable phase incidence capitals. With respect to the type of modulation to be used for the upcoming mobile communication, there is been healthy discussion happening with the standards to be used from the scientific community.

Here consumes remained an energetic conversation together, inside the technical public as well as inside calibrations, which inflection setup must be castoff aimed at the following cohort of mobile communiqué systems.

The $5 \mathrm{G}$ nets will innovator novel designs alike mist RAN also simulated RAN to ease a additional federal net setting up besides kind the finest usage of servers over contained data hubs on the net limits. Lastly, 5G will forefront the usage of reasoning wireless methods to let the substructure to routinely choose around the kind of station to be obtainable, distinguish amid mobile also secure substances, besides adapt to circumstances at an assumed period. In additional arguments, $5 \mathrm{G}$ nets will remain capable to oblige the manufacturing Internet also social net apps on the similar time.

Next-generation communication of mobile schemes require the expansion of wireless structures to encounter the novel supplies in subsequent net situations such by way of ultra- massive machine method communications in addition dependable improved mobile broadband.

Consequently the multi-carrier modulation takes remained extensively castoff; with its attractive appearances, MCM signifies the key constituent in wireless systems. A method that uses rectangular shaped pulses, generally known as OFDM is been more extensively used in systems of wireless communication.

A general MCM method is OFDM, that usages a foursided throb contour, also this method is prevalently castoff in wireless broad-band structures. OFDM has substantial limits, such by way of high OoB exemptions, which bases intrusive amid the adjacent stations, CP overhead, which origins a less efficacy, besides orthogonally, which needs severe spell also frequency management to elude incidence likewise efficacy offsets that kind it not the furthermost fit waveform aimed at all with submission situations.

The upcoming part of paper is been divided into section as below. In the next section explain about the research related topics, while the further section describe about the existing researches done on the topic. The next section to survey, we explain about the proposed methodology and we display the outcomes of the research in the results section. The last section we given the conclusion of the research added up with references.

\section{MAJOR TERMINOLOGY USED}

\section{A. Communication Technologies}

$2 \mathrm{G}$ is less for second-generation cellular net. $2 \mathrm{G}$ cellular nets remained commercially hurled going on GSM customary by Radiolinja in the 1991. 2G technologies permitted the numerous systems to deliver facilities such by way of text emails, image messages, as well as multimedia messages.

3G remains the 3rd group of wireless portable broadcastings expertise. It remains promotion for 2.5 G GPRS also 2.75 G EDGE nets, for quicker data transmission. This is grounded scheduled a customary of values used for portable strategies also mobile 
communications usage facilities also nets that obey by the International Mobile Telecommunications stipulations by the International Telecommunication Unification. 3G discoveries request in portable Internet access, wireless speech telephony, secure wireless Internet admittance, mobile television and video calls.

4th group of broad-band is $4 \mathrm{G}$ cellular net expertise, subsequent $3 \mathrm{G}$, also prior $5 \mathrm{G}$. A $4 \mathrm{G}$ structure necessity arrange for competences distinct by ITU over IMT Progressive. Conceivable also present requests comprise revised mobile net admittance, IP telephony, high-definition mobile TV, 3D television, gaming services and video conferencing,

In communications, LTE remains a normal for wireless broad-band communication aimed at portable diplomacies also data transmitters, grounded going on the GSM also HSPA expertise. It upsurges the scope besides speed by a dissimilar wireless border composed with core net developments. LTE stays the promotion trail for haulers by together GSM/UMTS nets also CDMA2000 nets. The dissimilar LTE incidences also ensembles castoff in diverse countries mean that solitary multi-band receivers remain clever to usage LTE over all nations wherever it is used.

Though previous peers of cellular expertise (such as $4 G$ LTE) attentive on endorsing connectivity, 5G receipts connectivity towards the subsequent glassy by bringing linked involvements since the mist to patrons. 5G nets are virtualized besides softwaredriven, also they adventure cloud expertise.

The 5G net resolve likewise abridge flexibility, with unified exposed wandering competences amid cellular also Wi-Fi admittance. Mobile handler's modulation sojourn associated by way of the interchange amid outside wireless networks besides wireless nets privileged structures deprived of operator interference or the necessity for manipulators towards reauthenticate.

\section{B. Communication Technologies}

In directive to diminish the multipath possessions, in its place of solitary carrier inflection, multicarrier tool is used wherever the complete setup is aligned into numerous minor bands. Now numerous multicarrier waveforms remain measured. OFDM which partakes remained by in $4 \mathrm{G}$ nevertheless owing to convinced disadvantages also ensures not demonstrate to stay a well-organized waveform for 5G.

i. OFDM:

The OFDM is a type of encoding in telecommunications, in which multiple frequency of the carrier wave are used to encode the data. In digital world, this OFDM has been developed in a vast in the field of digital communication which is been widely used for internet, television, radio broadcasting, mobile network communication, and various network communication fields.

ii. QAM:

Quadrature-amplitude-modulation (QAM) is the term of a group of digital modulation approaches and an associated group of analog variation approaches extensively castoff in recent telecommunications to communicate information. It carries dual analog communication signals, also known as dual digital bitstreams, by moderating the scales of both the carrier signals, by means of the amplitude variation similarity modulation method. The dual carrier signals of the similar frequency are away from the phase with every added by 90 degree, a modulation identified as quadrature. The transferred wave is generated by addition of two transporter signals together. On the receiver, both the signals can be coherently detached as of their orthogonally property. Additional key possessions is that inflections are with low band width signal forms likened to the carrier regularity, this is recognized as thin band supposition. 


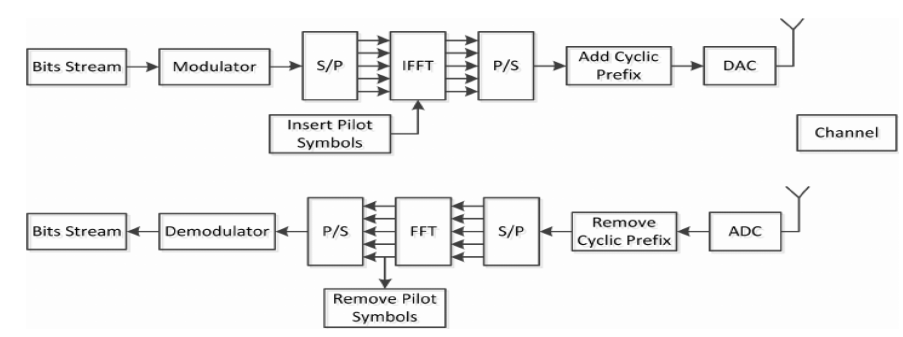

Figure 1. OFDM Block Diagram

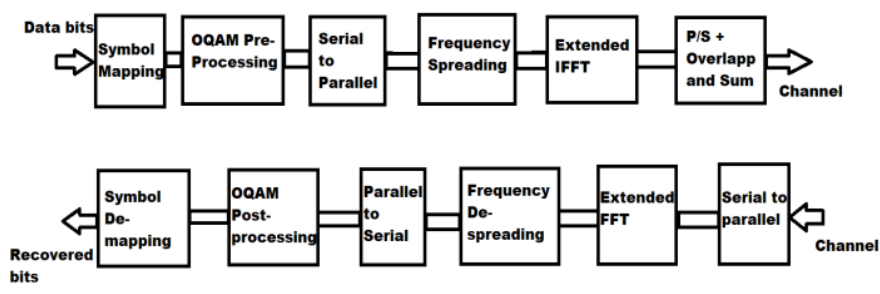

Figure 2. OFDM Block Diagram

\section{III.REVIEW PAPERS}

Heba et al [1] propose a pulse modelling filters in OQAM methods, directing to progress the scheme presentation. The evaluation of every pulse influential mesh in OQAM is calculated and associated other in relations of the PSD, signal-to-interference ratio, spectral efficiency, carrier frequency offset, time offset and BER performance concluded various stations. In addition, as multi input multi output is unique of the chief encounters related with FBMC, they propose novel method that custom the frequency based spreading founded on a Walshi Hadmard code on FBMC and examine the grouping of projected prototype pulse determining strainers with MIMO schemes.

Hadjer, et al [2], proposed a virtual revision of two structures, MIMO- FBMC/OQAM UFMC by dissimilar equalization methods such as ZF, MMSE in addition maximal ratio combining. These two inflection methods remain assessed over a Rayleigh station in terms of signal-to-noise ratio (SNR) also bit error ratio (BER). The simulation is achieved, also the precise examination indorses that the concert of the MIMO-FBMC / OQAM method by the MRC equalizer stays a improved superior than the MIMO-UFMC to overwhelm some inter symbol interference (ISI) besides diminishes the overall racket supremacy.
Dongkyu et al [3], examine the operative station also arise the signal-to-interference proportion aimed at stimulating white Gaussian noise stations in addition multipath fading stations. Grounded on the examination, the features of the actual station rendering to numerous sample colander signal also station replicas remain considered. Using the operative stations, we project two kinds of per-tone station equalizers also assess the while fault rate recitals by Monte Carlo imitations. Outcome of consequences demonstration that the FBMC-QAM coordination with two sample riddles Monte Carlo imitations attain nearly the similar bit inaccuracy rate recital as orthogonal incidence separation multiplexing by cyclic precede by accepting the signal-level per-tone station equalizer.

Ravindran et al [4], recommend the recurring precede over OFDM is castoff to diminish the multipath possessions also henceforth decrease ISI also ICI, nevertheless CP diminishes well-organized regular practice. Likewise the CP-OFDM partakes high OOB (Out Of Band) release by admiration to together side posses also consumes in elevation PAPR (Peak to Average Power Ratio). Consequently now, the two 5G waveforms UFMC also FBMC-OQAM remain deliberated which prepares not kind usage cyclic precede besides henceforth upsurges ethereal competence. Likewise these methods entail of surplus riddling procedure which modulation lessen $\mathrm{OOB}$ emission.

Jintaeet al [5], suggest a linearly similar to filter bank multicarrier (LP-FBMC) structure which services a quicker than Nyquist sampling towards removing inherent simulations. We signify the FBMC transceiver over a decade usage besides before smear singular value decomposition (SVD)- grounded linear alteration in command to adapt the overlay FBMC information into equivalent sovereign interferencefree information. This interference free distinguishing of the projected LP-FBMC structure allows the usage of conservative manifold feeler formations. Performance assessments illustration that out-of-band 
emission (OOBE) presentation of the projected LPFBMC structure is grander to individuals of conservative quadrature generosity established FBMC (FBMC-QAM) and orthogonal frequency division multiplexing (OFDM) structures.

Sanson et al [6], revised the detector dispensation of the OFDM indication to be pragmatic in a novel Filter Bank Multicarrier (FBMC) outline, which remains revealed to remain abundant grander to the OFDM scheme meanwhile it benevolences the similar bit error rate (BER) presentation in multipath stations, a abundant advanced ghostly competence also as exposed here, improved presentation in detector aiming. We assessed its enactment meant radar uses by combined communications.

\section{IV.PROPOSED METHOD}

Filter-bank-multicarrier (FBMC) stands an substitute broadcast technique that resolutions the overhead complications by means of in elevation excellence riddles that evade together admission besides egress sounds. Likewise, for of the precise squat out-of-band emanation of subcarrier riddles, request of FBMC over the uplink of multi user nets is inconsequential. It modulation be positioned deprived of bringing together of mobile handler protuberances signals.

\section{i. Transmitter}

In stint purview, the amount of multi carrier ciphers acquires overlay. The mandate of the sample riddle is designated as $2 * \mathrm{~K}-1$ wherever $\mathrm{K}$ represents be 2,3 or 4 , where Ts remains the sample period also ciphers $\mathrm{d}[\mathrm{n}]$ comprise one or else more info bits besides it could be multifaceted. The diagram of FBMC source is exposed in figure 3 underneath.

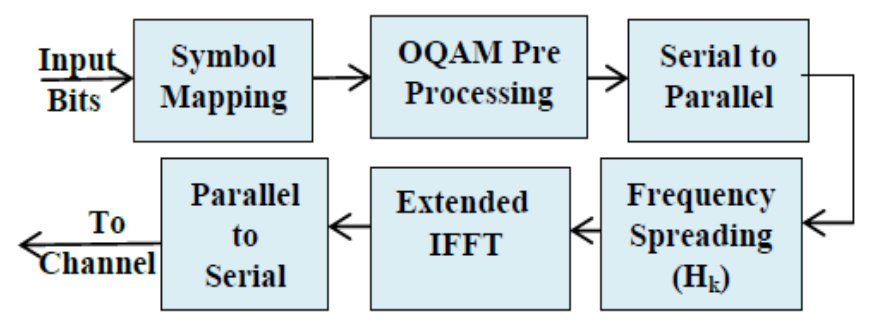

Figure 3. Block Diagram of FBMC Transmitter
Cutting-edge FBMC, the OQAM inflection also demodulation is castoff which conveys the information of actual also imaginary parts distinctly by a time offset interval of half of a symbol.

The FBMC modulation method usages a incidence dispersal ( $\mathrm{Hk}$ ) besides likewise usages $\mathrm{M}^{*} \mathrm{~J}$ distance IFFT cryptograms overlay through a interruption of $M / 2$, wherever $M$ is the amount of sub-haulers. OQAM remains active towards attain full station capacity. Concurrently the real besides imaginary portions of data cryptograms remain not conveyed also pretend part is overdue through partial the sign period. The enterprise of PHYDYAS prototype Constants is exposed underneath. The $\mathrm{N}$ subhauler incidence retort formula is:

$$
H(f)=\sum_{k}^{k-1}-(k-1) p_{k} \frac{\sin \left(\pi\left(f-\frac{k}{M k}\right) M k\right)}{M k \sin \left(\pi\left(f-\frac{k}{M k}\right)\right)^{\prime}}
$$

\section{ii. Receiver}

In the receiver, the information bits remain established after station formerly it adapts after serial to parallel demodulation. Cutting-edge FBMC multicarrier method, usages a net of sieves going on the flanks of reception ends, i.e. examination filters by a FFT by way of a Demodulator. Period arrangement amid the ciphers is meant thru T. The FBMC inflection method usages an incidence de-spreading likewise usages $\mathrm{M}^{*} \mathrm{~J}$ distance FFT ciphers met by an interruption of $M / 2$, wherever $M$ is the number of sub carriers. Concurrently the real in addition unreal portions of information ciphers remain not conventional also imaginary part remains behind thru half the figure extent. The figures ciphers remain over rehabilitated as of Parallel towards Serial adaptation. Aimed at OQAM post dispensation consumes the DEmultiplexing phases whish is for FBMCOQAM response achieves the actual to multifaceted number adaptation, de-staggering besides down sample. The dispensation takes coordinated filtering checked thru 
OQAM parting to become the conventional information ciphers. Then these customary codes are DE-mapped also ensuing bit error is very higher. DeMapper excerpts the whiles as of the moderated ciphers on the receiver. Consequently resultants jiffs remain improved by the receiver remain exposed over Figure 4.

Additional, we deliberate in what way FBMC modulation be used to proficiently sustenance altered use circumstances, intended for forthcoming wireless structures. We flinch by a description of the interval occurrence competence. Formerly, we combine the dual handlers by two dissimilar subcarrier spacing's portion, similar portions also compute the SIR.

$$
\mathrm{x}[\mathrm{n}]=\sum_{\mathrm{m}=-\infty}^{+\infty} \mathrm{x}_{\mathrm{R}}[\mathrm{n}] \mathrm{p}[\mathrm{n}-\mathrm{mN}]+\mathrm{x}_{\mathrm{I}}[\mathrm{n}] \mathrm{p}\left[\mathrm{n}-\mathrm{mN}-\frac{\mathrm{N}}{2}\right]
$$

Figure 4. FBMC Receiver Block Diagram

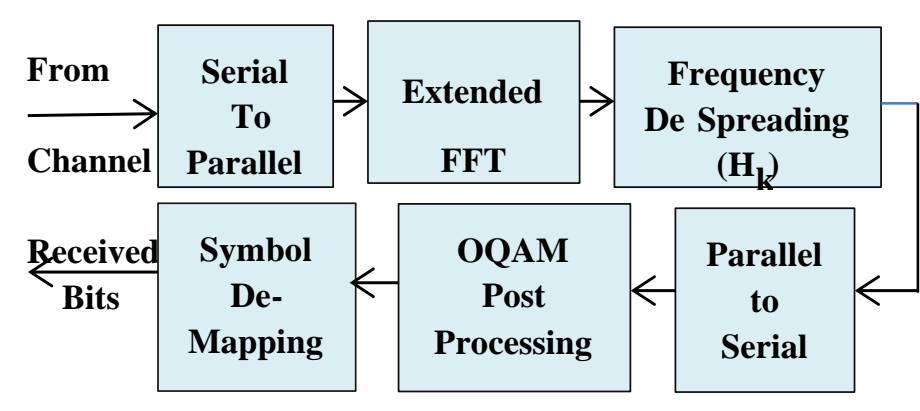

Figure 5. Time-Frequency Comparison

We equate the time-frequency competence of FBMCOQAM by that of $\mathrm{f}-\mathrm{OFDM}$. The sentinel period $\mathrm{T}$ G is elected consequently that $99.89 \%$ of the conveyed method, remains inside the interval interim KT + T G. Likewise, $99.99 \%$ of the conveyed oomph utilizing the PSD, remains in the bandwidth FL+F G.
Contingent arranged the precise use circumstance, one valor neediness to smear diverse brinks. Though, the rudimentary declarations will sojourn the similar. If solitary a scarce time-symbols remain castoff, for instance $\mathrm{K}=1$ for $\mathrm{f}-\mathrm{OFDM}$ also $\mathrm{K}=2$ aimed at FBMC, f-OFDM exhibitions improved error than FBMC owing to a superior sentinel period obligatory over FBMC (solitary if no overlying amid chunks is likely). About $\mathrm{K}=5$ multifaceted time-symbols ( $\mathrm{K}=10$ realsymbols) remain obligatory to kind the timefrequency competence of FBMC improved that of $f-$ OFDM, though this be contingent sturdily arranged the amount of subcarriers. Once the amount of timesymbols tactics eternity, which is about actual in numerous circumstances since chunks (subframes) modulation effortlessly overlay, solitary OOB releases remain relevant likewise FBMC strongly exceeds $f$ OFDM. Formerly $\mathrm{K}=15$ multifaceted time-symbols remain adequate to originate near toward the boundary of $\mathrm{K} \rightarrow \infty$ aimed on the Hermit pulse (95\% threshold); aimed at the PHYDYAS filter it is $\mathrm{K}=30$.

\section{RESULTS}

In this section we use few parameter to compare the proposed and existing techniques with respect to the comparative parameters.

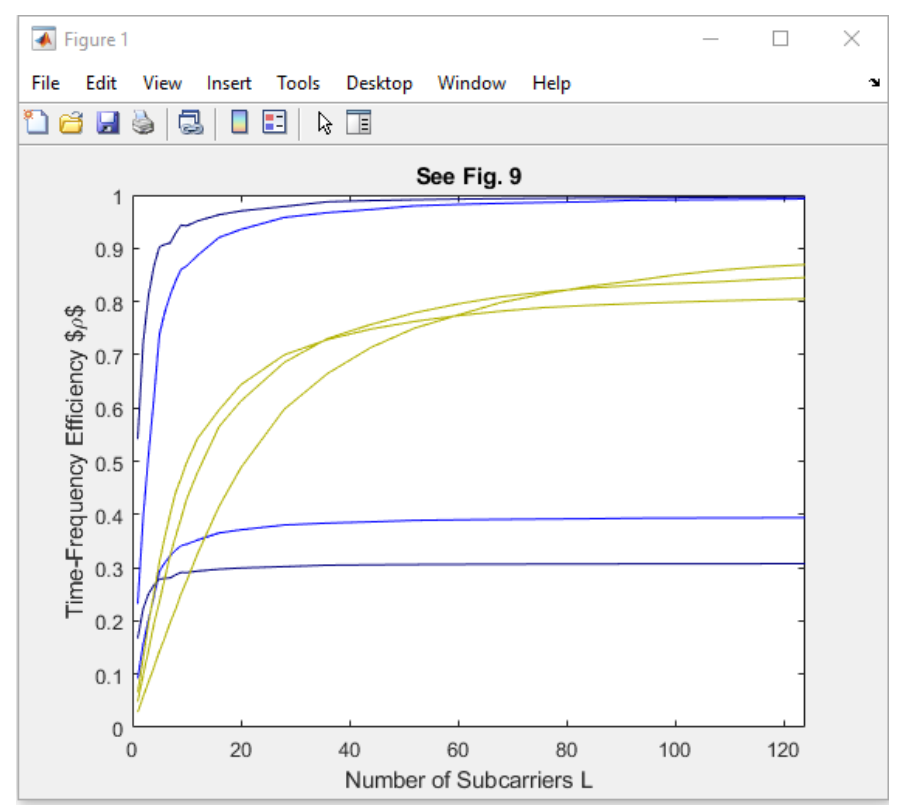


i. PSD

The PSD be contingent on the firmness of the DAC $(\mathrm{NFFT}=1024)$. Quantization noise makes to comparatively high OOB emanations. Though, altogether those errors remain not boundless toward FBMC nevertheless likewise seem in windowed also filtered OFDM. Consequently, the perception of shrill digital riddles to permit a supple time-frequency provision, figure 5 solitary workings aimed at a adequately lined influence amp also a satisfactorily high DAC resolve. If these circumstances remain not attained, we remain relay towards less depend analog filters.

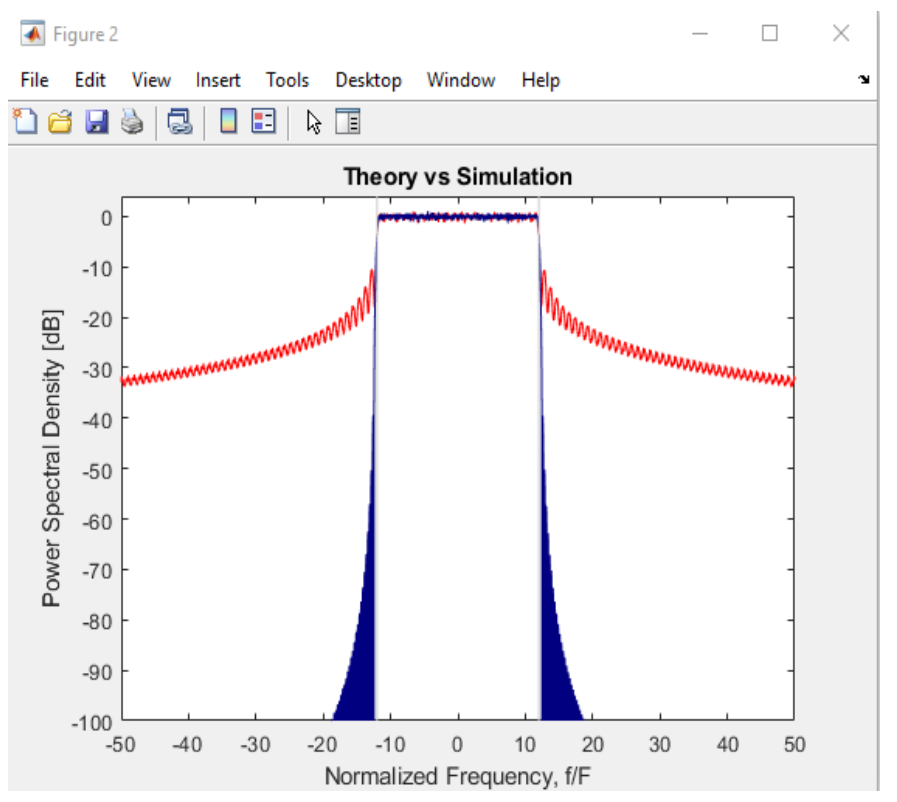

Figure 5. PSD comparison

\section{ii. BER over SNR:}

Figure 6 demonstrations the fallouts of real-world tested amounts obtainable, aimed at outdoor-toindoor broadcasts on a carrier frequency incidence of $2.5 \mathrm{GHz}$ also aimed at indoor-to-indoor broadcasts at a hauler incidence of $60 \mathrm{GHz}$. Together, FBMC besides OFDM, have the similar Bit Error Ratio (BER), authenticating the dispersal method. Nevertheless, FBMC consumes abundant improved ethereal possessions.

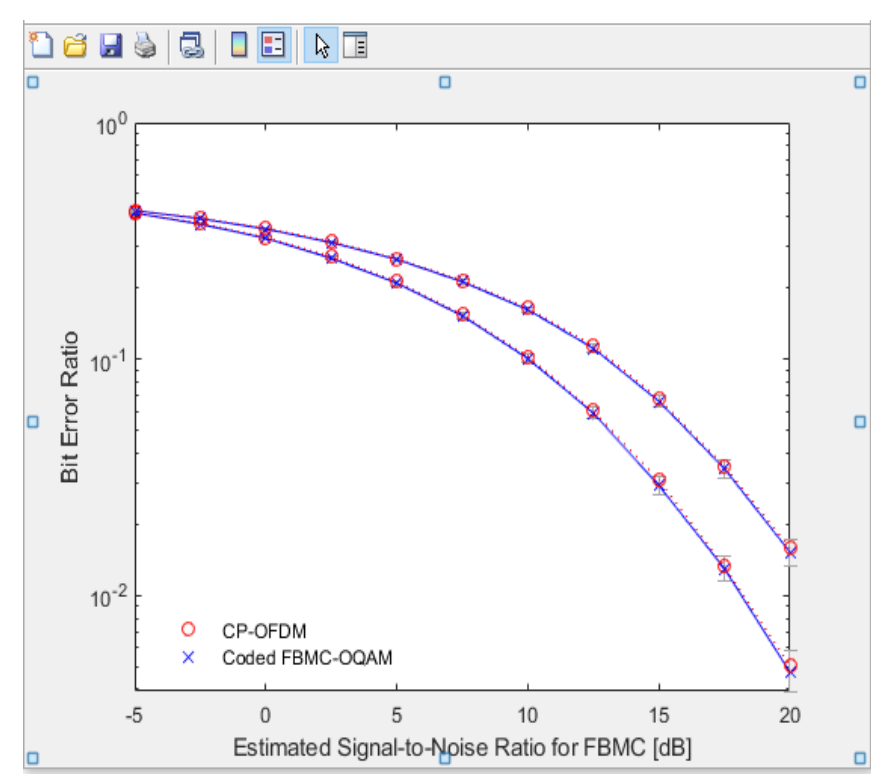

\section{iii. Sub-Carrier Spacing}

We must retain in attention that, without of obtain filtering also riddling, the intrusion as of handler 1 to handler 2 is sophisticated than the intrusion after operative 2 to operative 1 , which ampule remain inferred after Figure 7. Formerly we smear windowing besides sieving on the receiver, together users' capability almost the similar intrusion power.

Figure 6. BER versus SNR comparison

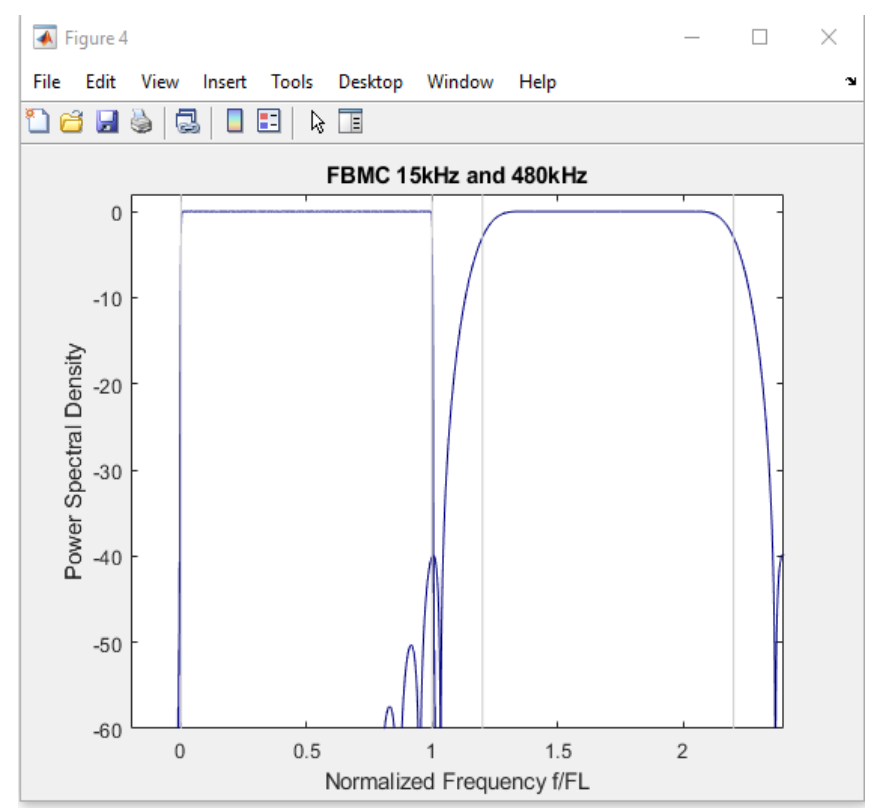

Figure 7. Sub-Carrier spacing Comparison 


\section{CONCLUSION}

If the number of sub-carriers are high, in OFDM related modulation techniques such as UFMC, $\mathrm{f}$ OFDM, WOLA we get a higher spectral efficiency. But in the future wireless communication systems we have not be able to see these sub carrier frequency's in all the modulation techniques. The OFDM efficiency is very much better than the FBMC modulation technique if the sub carrier frequency are small. With the proposed methodology, we can overcome lot problems that occur in the FBMC during the MIMO, channel error loss and mid-frequency losses.

\section{REFERENCES}

[1] H. M. Abdel-Atty, W. A. Raslan and A. T. Khalil, "Evaluation and Analysis of FBMC/OQAM Systems Based on Pulse Shaping Filters," in IEEE Access, vol. 8, pp. 55750-55772, 2020, doi: 10.1109/ACCESS.2020.2981744.

[2] B. Hadjer and B. Abdelhafid, "Comparison \& Performance Evaluation of MIMO-FBMC and MIMO-UFMC systems for various equalization techniques," 2019 International Conference on Networking and Advanced Systems (ICNAS), 2019, pp. 1-5, doi: 10.1109/ICNAS.2019.8807861.

[3] D. Sim and C. Lee, "Performance Evaluation Based on Effective Channel Analysis for FBMC-QAM System With Two Prototype Filters," in IEEE Transactions on Communications, vol. 67, no. 5, pp. 3552-3565, May 2019, doi: 10.1109/TCOMM.2019.2893316.

[4] R. Ravindran and A. Viswakumar, "Performance evaluation of $5 \mathrm{G}$ waveforms: UFMC and FBMC-
OQAM with Cyclic Prefix-OFDM System," 2019 9th International Conference on Advances in Computing and Communication (ICACC), 2019, pp. 6-10, doi: 10.1109/ICACC48162.2019.8986195.

[5] J. Kim, Y. Park, S. Weon, J. Jeong, S. Choi and D. Hong, "A New Filter-Bank Multicarrier System: The Linearly Processed FBMC System," in IEEE Transactions on Wireless Communications, vol. 17, no. 7, pp. 4888-4898, July 2018, doi: 10.1109/TWC.2018.2832646.

[6] J. Sanson, A. Gameiro, D. Castanheira and P. P. Monteiro, "24 GHz QAM-FBMC Radar with Communication System (RadCom)," 2018 AsiaPacific Microwave Conference (APMC), 2018, pp. 678-680, doi: 10.23919/APMC.2018.8617314.

[7] P. Popovski, K. F. Trillingsgaard, O. Simeone, and G. Durisi, "5G wireless network slicing for eMBB, URLLC, and mMTC: A communication theoretic view," IEEE Access, vol. 6, pp. 55765-55779, 2018.

[8] S. Taheri, M. Ghoraishi, P. Xiao, and L. Zhang, "Efficient implementation of filter bank multicarrier systems using circular fast convolution," IEEE Access, vol. 5, pp. 2855-2869, 2017.

\section{Cite this article as :}

Dr. Shankaraiah, Tejas K B , "Performance Evaluation of FBMC for Future Communication", International Journal of Scientific Research in Science, Engineering and Technology (IJSRSET), Online ISSN : 2394-4099, Print ISSN : 2395-1990, Volume 8 Issue 4, pp. 199-206, July-August 2021. Available at doi $\quad$ : https://doi.org/10.32628/IJSRSET218438 Journal URL : https://ijsrset.com/IJSRSET218438 